\title{
ON A CLASS OF MEROMORPHIC STARLIKE FUNCTIONS WITH NEGATIVE COEFFICIENTS
}

\author{
K. K. DIXIT AND S. K. PAL
}

Abstract. Let $T_{M}^{*}\left(A, B, z_{0}\right)$ denote the class of functions $f(z)=\frac{a}{z}-\sum_{n=1}^{\infty} a_{n} z^{n}$ $\left(a \geq 1, a_{n} \geq 0\right)$ regular and univalent in unit disc $U^{\prime}=\{z: 0<|z|<1\}$, satisfying the condition

$$
-z \frac{f^{\prime}(z)}{f(z)}=\frac{1+A w(z)}{1+B w(z)}, \quad \text { for } z \in U^{\prime} \text { and } w \in E
$$

(where $E$ is the class of analytic functions $w$ with $w(0)=0$ and $|w(z)| \leq 1$ ), where $-1 \leq A<B \leq 1,0 \leq B \leq 1$ and $f\left(z_{0}\right)=\frac{1}{z_{0}}\left(0<z_{0}<1\right)$. In this paper sharp coefficient estimates, distortion properties and radius of meromorphic convexity for functions in $T_{M}^{*}\left(A, B, z_{0}\right)$ have been obtained. We also study integral transforms of functions in $T_{M}^{*}\left(A, B, z_{0}\right)$. In the last, it is proved that the class $T_{M}^{*}\left(A, B, z_{0}\right)$ is closed under convex linear combinations.

\section{Introduction}

Let $S$ denote the class of functions of the form $f(z)=z+\sum_{n=2}^{\infty} a_{n} z^{n}$ that are analytic in $U=\{z:|z|<1\}$. Denote by $S^{*}(\rho)$ and $K(\rho),(0 \leq \rho<1)$ the subclass of functions $f$ in $S$ that satisfy respectively the conditions:

$$
\operatorname{Re}\left[z \frac{f^{\prime}(z)}{f(z)}\right]>\rho \text { and } \operatorname{Re}\left[1+\frac{z f^{\prime \prime}(z)}{f^{\prime}(z)}\right]>\rho \quad \text { for } z \in U .
$$

Functions in $S^{*}(\rho)$ and $K(\rho)$ are called starlike functions of order $\rho$ and convex functions of order $\rho$ respectively.

Let $T$ denote the subclass of functions in $S$ of the form:

$$
f(z)=z-\sum_{n=2}^{\infty} a_{n} z^{n}, a_{n} \geq 0 . \quad \text { Also set } T^{*}(\rho)=T \cap S^{*}(\rho) \text { and } C(\rho)=T \cap K(\rho) .
$$

Received April 18, 1993; revised Octember 3, 1994. 
The classes $T^{*}(\rho)$ and $C(\rho)$ possess some very interesting properties and have been studied in detail by Silverman $[9,11]$. The extreme points for prestar-like functions having negative coefficients have been determined by Silverman and Silvia [12]. In this paper coefficient, distortion and radii of univalence starlikeness and convexity theorem have also been obtained.

Let $T^{*}(A, B, K)$ be the class of functions $f(z)=a_{1} z-\sum_{n=k}^{\infty}\left|a_{n}\right|_{z} n\left(a_{1}>0, K \geq 2\right)$ regular and univalent in the unit disc $U=\{z:|z|<1\}$ and satisfying $\mid\left\{\left(z f^{\prime}(z) / f(z)\right)-\right.$ $1\} /\left\{A-B z f^{\prime}(z) / f(z)\right\} \mid<1, z \in U$, Where $-1 \leq B<A \leq 1$ and $-1 \leq B \leq 0$. Let $0<z_{0}<1$, Kumar [4] denoted by $T_{1}^{*}\left(A, B, K, z_{0}\right)$ and $T_{2}^{*}\left(A, B, K, z_{0}\right)$, two subclasses of $T^{*}(A, B, K)$, consisting of functions which satisfy $f\left(z_{0}\right)=z_{0}$ and $f^{\prime}\left(z_{0}\right)=1$ respectively. Kumar [4] has obtained many results including coefficient estimates, distortion and closure theorems and radius of convexity of order $\rho(0 \leq \rho<1)$ for the classes $T_{1}^{*}\left(A, B, K, z_{0}\right)$ and $T_{2}^{*}\left(A, B, K, z_{0}\right)$.

Two subclasses obtained by replacing $z f^{\prime}(z) / f(z)$ by $f^{\prime}(z) / a_{1}$ in the definitions of $T_{m}^{*}\left(A, B, K, z_{0}\right), m=1,2$ have been studied by Kumar and Shukla [5].

Let $\sum$ denote the class of functions of the form:

$$
g(z)=\frac{1}{z}+\sum_{n=1}^{\infty} a_{n} z^{n}
$$

which are regular in $U^{\prime}=\{z: 0<|z|<1\}$ having a simple pole at the origin. Let $\sum_{s}$ denote the class of functions in $\sum$ which are univalent in $U^{\prime}$ and $\sum^{*}(\rho)$ and $\sum_{k}(\rho)$ $(0 \leq \rho<1)$ be the subclasses of functions $f$ in $\sum$ satistying repectively the conditions:

$$
R_{e}\left\{-z \frac{f^{\prime}(z)}{f(z)}\right\}>\rho
$$

and

$$
R e\left[-\left\{1+\frac{z f^{\prime \prime}(z)}{f^{\prime}(z)}\right\}\right]>\rho \quad \text { for } \quad z \in U^{\prime} .
$$

Functions in $\Sigma^{*}(\rho)$ and $\sum_{k}(\rho)$ are called meromorphically starlike functions of order $\rho$ and meromorphically convex functions of order $\rho$ respectively.

The classes $\sum^{*}(\rho)$ and $\sum_{k}(\rho)$ have been extensively studied by Pommerinke [7], Clunie [1], Kazmarski [3], Royster [8] and others.

Since to some extent the work in univalent meromorphic case has paralleled to that of regular univalent case, one is interested to investigate for a class of functions which are regular in $U^{\prime}$ with simple pole at the origin having properties analogous to those of $T^{*}(A, B, K)$. To this end we introduce in this section such a class of functions which are regular in $U^{\prime}$ and which have the properties simillar to those of $T^{*}(A, B, K)$.

Let $T_{M}^{*}$ denote the class of functions $f(z)=\frac{a}{z}-\sum_{n=1}^{\infty} a_{n} z^{n}\left(a \geq 1, a_{n} \geq 0\right)$, (The condition $a \geq 1$ is necessary, see Nihari $[6$, ex. 8, p. 238]) regular and univalent in the 
$\operatorname{disc} U^{\prime}$. Let $T_{M}^{*}(A, B)$ denote the subclass of function in $T_{M}^{*}$ satisfying the condition

$$
-z \frac{f^{\prime}(z)}{f(z)}=\frac{1+A w(z)}{1+B w(z)}, \text { for } z \in U^{\prime}, w \in E,
$$

where $-1 \leq A<B \leq 1,0 \leq B \leq 1$. Also $T_{m}^{*}\left(A, B, z_{0}\right)$ denote the subclass of function in $T_{M}^{*}(A, B)$ satisfying $f\left(z_{0}\right)=\frac{1}{z_{0}}$ (where $0<z_{0}<1$ ).

The present chapter is devoted to obtain sharp coefficient estimates, distortion properties and radius of meromorphic convexity for functions in $T_{M}^{*}\left(A, B, z_{0}\right)$. We study integral transforms of functions in $T_{M}^{*}\left(A, B, z_{0}\right)$. In the last it is shown that the class $T_{M}^{*}\left(A, B, z_{0}\right)$ is closed under convex linear combinations.

\section{Main Results}

First we prove an important theorem which is to be used in next coming theorems.

Theorem 2.1. Let $f(z)=\frac{1}{z}-\sum_{n=1}^{\infty}\left|a_{n}\right| z^{n}$ be regular in $U^{\prime}$ and belongs in $T_{M}^{*}(A, B)$ if and only if

$$
\sum_{n=1}^{\infty}\{n(1-B)+1-A\}\left|a_{n}\right| \leq(B-A)
$$

Proof. Consider the expression

$$
H\left(f, f^{\prime}\right)=\left|z f^{\prime}(z)+f(z)\right|-\left|B z f^{\prime}(z)+A f(z)\right| .
$$

Replacing $f$ and $f^{\prime}$ by their series expansions we have, for $0<|z|=r<1$

$$
\begin{aligned}
H\left(f, f^{\prime}\right) & =\left|\sum_{n=1}^{\infty}(n+1)\right| a_{n}\left|z^{n}\right|-\left|(A-B) \cdot \frac{1}{z}-\sum_{n=1}^{\infty}(A+B n)\right| a_{n}\left|z^{n}\right| \\
& \leq\left|\sum_{n=1}^{\infty}(n+1)\right| a_{n}\left|z^{n}\right|-\left[\sum_{n=1}^{\infty}(A+B n)\left|a_{n}\right| z^{n}-\left|(A-B) \frac{1}{z}\right|\right] \\
& =\sum_{n=1}^{\infty}(n+1)\left|a_{n}\right||z|^{n}-\sum_{n=1}^{\infty}(A+B n)\left|a_{n}\right||z|^{n}+(A-B) \frac{1}{|z|}
\end{aligned}
$$

or

$$
r H\left(f, f^{\prime}\right) \leq \sum_{n=1}^{\infty}\{n(1-B)+1-A\}\left|a_{n}\right| r^{n+1}+(A-B) .
$$

Since this holds for all $r, 0<r<1$, making $r \rightarrow 1$, we have

$$
H\left(f, f^{\prime}\right) \leq \sum_{n=1}^{\infty}\{n(1-B)+1-A\}\left|a_{n}\right|+(A-B) \leq 0
$$


in view of (2.1). From (2.2), we thus have

$$
\left|\frac{z \frac{f^{\prime}(z)}{f(z)}+1}{B z \frac{f^{\prime}(z)}{f(z)}+A}\right| \leq 1
$$

Hence $f \in T_{M}^{*}(A, B)$.

Conversely, let $f(z)=\frac{1}{z}-\sum_{n=1}^{\infty}\left|a_{n}\right| z^{n}, a_{n} \geq 0$ is in $T_{M}^{*}(A, B)$, i.e.

$$
\left|\frac{z \frac{f^{\prime}(z)}{f(z)}+1}{B z \frac{f^{\prime}(z)}{f(z)}+A}\right| \leq 1
$$

or

$$
\left|\frac{\sum_{n=1}^{\infty}(n+1)\left|a_{n}\right| z^{n+1}}{(B-A)+\sum_{n=1}^{\infty}(A+B n)\left|a_{n}\right| z^{n+1}}\right| \leq 1
$$

Since $\operatorname{Re}(z) \leq|z|$

$$
\operatorname{Re}\left\{\frac{\sum_{n=1}^{\infty}(n+1)\left|a_{n}\right| z^{n+1}}{(B-A)+\sum_{n=1}^{\infty}(A+B n)\left|a_{n}\right| z^{n+1}}\right\} \leq 1 .
$$

choosing $z=r$ with $0<r<1$, we get

$$
\frac{\sum_{n=1}^{\infty}(n+1)\left|a_{n}\right| r^{n+1}}{(B-A)+\sum_{n=1}^{\infty}(A+B n)\left|a_{n}\right| r^{n+1}} \leq 1 .
$$

Let $S(r)=(B-A)+\sum_{n=1}^{\infty}(A+B n)\left|a_{n}\right| r^{n+1}, S(r) \neq 0$ for $0<r<1, S(r)>0$ for sufficiently small values of $r$ and $S(r)$ is continuous for $0<r<1$. Hence $S(r)$ can not be negative for any value of $r$ such that $0<r<1$. Upon clearing the denominator in (2.3) and letting $r \rightarrow 1$ we get

$$
\sum_{n=1}^{\infty}(n+1)\left|a_{n}\right| \leq(D-A)+\sum_{n=1}^{\infty}(A+B n)\left|a_{n}\right|
$$

or

$$
\sum_{n=1}^{\infty}\{n(1-B)+1-A\}\left|a_{n}\right| \leq B-A .
$$

Hence the theorem. 
Theorem 2.2. Let $f(z)=\frac{a}{z}-\sum_{n=1}^{\infty}\left|a_{n}\right| z^{n}$ (where $a \geq 1$ ). If $f$ is regular in $U$ and satisfies $f\left(z_{0}\right)=\frac{1}{z_{0}}$, then $f \in T_{M}^{*}\left(A, B, z_{0}\right)$ if and only if

$$
\sum_{n=1}^{\infty}\left[\{n(1-B)+1-A\}-(B-A) z_{0}^{n+1}\right]\left|a_{n}\right| \leq B-A .
$$

The result is sharp.

Proof. We know from theorem 2.1 that a function $g(z)=\frac{1}{z}-\sum_{n=1}^{\infty}\left|b_{n}\right| z^{n}$ regular in $U$, satisfies

$$
\left|\frac{z \frac{g^{\prime}(z)}{g(z)}+1}{B z \frac{g^{\prime}(z)}{g(z)}+A}\right|<1, z \in U
$$

if and only if

$$
\sum_{n=1}^{\infty}\{n(1-B)+1-A\}\left|b_{n}\right| \leq B-A .
$$

Applying that result to the function $g(z)=f(z) / a$, we find that $f$ satisfies (1.1) if and only if

$$
\sum_{n=1}^{\infty}\{n(1-B)+1-A\}\left|a_{n}\right| \leq(B-A) a .
$$

Since $f\left(z_{0}\right)=\frac{1}{z_{0}}$, we also have from the representation of $f(z)$ that

$$
a=1+\sum_{n=1}^{\infty}\left|a_{n}\right| z_{0}^{n+1}
$$

Putting this value of a in the above inequality we obtain the required result

$$
\sum_{n=1}^{\infty}\left[\{n(1-B)+1-A\}-(B-A) z_{0}^{n+1}\right]\left|a_{n}\right| \leq B-A .
$$

Sharpness follows if we take the extremal function

$$
f(z)=\frac{\{n(1-B)+1-A\} \frac{1}{z}-(B-A) z^{n}}{\{n(1-B)+1-A\}-(B-A) z_{0}^{n+1}}, n=1,2, \ldots
$$

Theorem 2.3. $f \in T_{M}^{*}\left(A, B, z_{0}\right)$, then $f$ is meromorphically convex of order $\delta(0 \leq \delta<1)$ in the disc $|z|<R$, where

$$
R=\inf _{n>1}\left[\frac{(1-\delta)\{n(1-B)+1-A\}}{n(n+\delta)(B-A)}\right]^{1 /(n+1)}
$$


The result is sharp with extremal function (2.7).

Proof. In order to establish the required result, it suffices to show that

$$
\left|2+\frac{z f^{\prime \prime}(z)}{f^{\prime}(z)}\right| \leq 1-\delta
$$

or

$$
\left|\frac{f^{\prime}(z)+\left[z f^{\prime}(z)\right]^{\prime}}{f^{\prime}(z)}\right| \leq 1-\delta
$$

and

$$
\left|\frac{f^{\prime}(z)+\left[z f^{\prime}(z)\right]^{\prime}}{f^{\prime}(z)}\right| \leq \frac{\sum_{n=1}^{\infty} \frac{n(n+1)}{a}\left|a_{n}\right||z|^{n+1}}{1+\sum_{n=1}^{\infty} \frac{n}{a}\left|a_{n}\right||z|^{n+1}} .
$$

This will be bounded by $(1-\delta)$ if

$$
\sum_{n=1}^{\infty} n(n+\delta)\left|a_{n}\right||z|^{n+1} \leq a(1-\delta) .
$$

Since $a=1+\sum_{n=1}^{\infty}\left|a_{n}\right| z_{0}^{n+1}$, the above inequality can be written as

$$
\sum_{n=1}^{\infty} \frac{\left[n(n+\delta)|z|^{n+1}-(1-\delta) z_{0}^{n+1}\right]}{1-\delta}\left|a_{n}\right| \leq 1
$$

Also by Theorem 2.2, we have

$$
\sum_{n=1}^{\infty} \frac{\{n(1-B)+1-A\}-(B-A) z_{0}^{n+1}}{(B-A)}\left|a_{n}\right| \leq 1
$$

Hence (2.8) will be satisfied if

$$
\frac{n(n+\delta)|z|^{n+1}-(1-\delta) z_{0}^{n+1}}{1-\delta} \leq \frac{\{n(1-B)+1-A\}-(B-A) z_{0}^{n+1}}{(B-A)}
$$

or

$$
|z|<\left[\frac{(1-\delta)\{n(1-B)+1-A\}}{n(n+\delta)(B-A)}\right]^{1 /(n+1)},
$$

for each $n=1,2, \ldots$. This completes the proof of theorem.

Theorem 2.4. If $f \in T_{M}^{*}\left(A, B, z_{0}\right)$, then the integral transform

$$
F(z)=c \int_{0}^{1} u^{c} f(u z) d u, \text { for } 0<c<\infty
$$


is in $T_{M}\left(A^{\prime}, B^{\prime}, z_{0}\right)$, where

$$
\frac{1-B^{\prime}}{B^{\prime}-A^{\prime}} \leq \frac{(2-A-B)(c+2)-(B-A) c}{2 c(B-A)}-\frac{z_{0}^{2}}{c}
$$

The result is sharp for the extremal function

$$
f(z)=\frac{(2-A-B) \frac{1}{z}-(B-A) z}{(2-A-B)-(B-A) z_{0}^{2}} .
$$

Proof. Suppose $f(z)=\frac{a}{z}-\sum_{n=1}^{\infty}\left|a_{n}\right| z^{n} \in T_{M}\left(A, B, z_{0}\right)$, then

$$
\begin{aligned}
F(z) & =c \int_{0}^{1} u^{c}\left[\frac{a}{u z}-\sum_{n=1}^{\infty}\left|a_{n}\right|\left(u^{n} z^{n}\right)\right] d u \\
& =c \int_{0}^{1}\left[u^{c-1} \frac{a}{z}-\sum_{n=1}^{\infty}\left|a_{n}\right| z^{n} u^{n+c}\right] d u \\
& =c\left[\frac{u^{c}}{c} \frac{a}{z}-\sum_{n=1}^{\infty}\left|a_{n}\right| z^{n} \frac{u^{n+c+1}}{(n+c+1)}\right]_{0}^{1} \\
& =c\left[\frac{a}{c z}-\sum_{n=1}^{\infty} \frac{\left|a_{n}\right|}{(n+c+1)} z^{n}\right] \\
& =\frac{a}{z}-\sum_{n=1}^{\infty} \frac{c}{(n+c+1)}\left|a_{n}\right| z^{n} .
\end{aligned}
$$

It is sufficient to show that

$$
\sum_{n=1}^{\infty} \frac{\left[\left\{n\left(1-B^{\prime}\right)+1-A^{\prime}\right\}-\left(B^{\prime}-A^{\prime}\right) z_{0}^{n+1}\right]}{\left(B^{\prime}-A^{\prime}\right)(n+c+1)}\left|a_{n}\right| \leq 1
$$

Since $f \in T_{M}\left(A, B, z_{0}\right)$, it implies that

$$
\sum_{n=1}^{\infty} \frac{\{n(1-B)+1-A\}-(B-A) z_{0}^{n+1}}{(B-A)}\left|a_{n}\right| \leq 1 .
$$

(2.9) will be satisfied if

$$
\begin{gathered}
\frac{\left[\left\{n\left(1-B^{\prime}\right)+1-A^{\prime}\right\}-\left(B^{\prime}-A^{\prime}\right) z_{0}^{n+1}\right] c}{\left(B^{\prime}-A^{\prime}\right)(n+c+1)} \leq \frac{\{n(1-B)+1-A\}-(B-A) z_{0}^{n+1}}{(B-A)} \\
\quad \text { for each } n, \\
\frac{n\left(1-B^{\prime}\right)+1-A^{\prime}}{B^{\prime}-A^{\prime}} \leq \frac{\{n(1-B)+1-A\}(n+c+1)}{(B-A) c}-\frac{(n+1)}{c} z_{0}^{n+1},
\end{gathered}
$$


or

$$
\frac{1-B^{\prime}}{B^{\prime}-A^{\prime}} \leq \frac{\{n(1-B)+1-A\}(n+c+1)-(B-A) c}{(B-A)(n+1) c}-\frac{1}{c} z_{0}^{n+1}
$$

The right hand side of (2.10) is an increasing function of $n$, therefore putting $n=1$ in (2.10) we get

$$
\frac{1-B^{\prime}}{B^{\prime}-A^{\prime}} \leq \frac{(2-A-B)(c+2)-(B-A) c}{2 c(B-A)}-\frac{z_{0}^{2}}{c}
$$

Hence the theorem.

Theorem 2.5. Let $\gamma$ be a real number such that $\gamma>1$. If $f \in T_{M}^{*}\left(A, B, z_{0}\right)$, then the function $F$ defined by

$$
F(z)=\frac{(\gamma-1)}{z^{\gamma}} \int_{0}^{z} t^{\gamma-1} f(t) d t
$$

also belongs to $T_{M}^{*}\left(A, B, z_{0}\right)$.

Proof. Let $f(z)=\frac{a}{z}-\sum_{n=1}^{\infty}\left|a_{n}\right| z^{n}$. Then from the representation of $F(z)$, it follows that

$$
\begin{aligned}
F(z) & =\frac{(\gamma-1)}{z^{\gamma}} \int_{0}^{z} t^{\gamma-1}\left[\frac{a}{t}-\sum_{n=1}^{\infty}\left|a_{n}\right| t^{n}\right] d t \\
& =\frac{\gamma-1}{z^{\gamma}} \int_{0}^{z}\left[a t^{\gamma-2}-\sum_{n=1}^{\infty}\left|a_{n}\right| t^{n+\gamma-1}\right] d t \\
& =\frac{\gamma-1}{z^{\gamma}}\left[a \frac{t^{\gamma-1}}{\gamma-1}-\sum_{n=1}^{\infty}\left|a_{n}\right| \frac{t^{n+\gamma}}{n+\gamma}\right]_{0}^{z} \\
& =\frac{\gamma-1}{z^{\gamma}}\left[a \frac{z^{\gamma-1}}{\gamma-1}-\sum_{n=1}^{\infty} \frac{\left|a_{n}\right|}{n+\gamma} z^{n+\gamma}\right] \\
& =\frac{a}{z}-\sum_{n=1}^{\infty} \frac{\gamma-1}{n+\gamma}\left|a_{n}\right| z^{n}
\end{aligned}
$$

or

$$
F(z)=\frac{a}{z}-\sum_{n=1}^{\infty}\left|b_{n}\right| z^{n}
$$


where $\left|b_{n}\right|=\frac{\gamma-1}{n+\gamma}\left|a_{n}\right|$. Therefore,

$$
\begin{aligned}
& \sum_{n=1}^{\infty}\left[\{n(1-B)+1-A\}-(B-A) z_{0}^{n+1}\right]\left|b_{n}\right| \\
= & \sum_{n=1}^{\infty}\left[\frac{\gamma-1}{n+\gamma}\right]\left[\{n(1-B)+1-A\}-(B-A) z_{0}^{n+1}\right]\left|a_{n}\right| \\
\leq & \sum_{n=1}^{\infty}\left[\{n(1-B)+1-A\}-(B-A) z_{0}^{n+1}\right]\left|a_{n}\right| \\
\leq & (B-A), \text { by Theorem } 2.2 .
\end{aligned}
$$

Hence $F \in T_{M}^{*}\left(A, B, z_{0}\right)$. this completes the proof of the theorem.

Theorem 2.6. Let $f_{j}(z)=\frac{a_{j}}{z}-\sum_{n=1}^{\infty}\left|a_{n j}\right| z^{n}, j=1,2, \ldots, m$. If $f_{j} \in T_{M}^{*}\left(A, B, z_{0}\right)$ for each $j=1,2, \ldots, m$, then the function

$$
h(z)=\frac{b}{z}-\sum_{n=1}^{\infty}\left|b_{n}\right| z^{n}
$$

also belongs to $T_{M}^{*}\left(A, B, z_{0}\right)$ where

$$
\begin{aligned}
b & =\sum_{j=1}^{m} \lambda_{j} a_{j},\left|b_{n}\right|=\sum_{j=1}^{m} \lambda_{j}\left|a_{n j}\right| \quad(n=1,2, \ldots, m), \\
\lambda_{j} & \geq 0 \text { and } \sum_{j=1}^{m} \lambda_{j}=1
\end{aligned}
$$

Proof. Since $f_{j} \in T_{M}^{*}\left(A, B, z_{0}\right)$, then

$$
\sum_{n=1}^{\infty}\left[\{n(1-B)+1-A\}-(B-A) z_{0}^{n+1}\right]\left|a_{n j}\right| \leq B-A, \quad j=1,2, \ldots, m .
$$

Therefore,

$$
\begin{aligned}
& \sum_{n=1}^{\infty}\left[\{n(1-B)+1-A\}-(B-A) z_{0}^{n+1}\right]\left|b_{n}\right| \\
= & \sum_{n=1}^{\infty}\left[\{n(1-B)+1-A\}-(B-A) z_{0}^{n+1}\right] \sum_{j=1}^{m} \lambda_{j}\left|a_{n j}\right| \\
= & \sum_{j=1}^{m} \lambda_{j} \sum_{n=1}^{\infty}\left[\{n(1-B)+1-A\}-(B-A) z_{0}^{n+1}\right]\left|a_{n j}\right| \\
\leq & \sum_{j=1}^{m} \lambda_{j}(B-A)=(B-A) .
\end{aligned}
$$


Hence by Theorem $2.2, h \in T_{M}^{*}\left(A, B, z_{0}\right)$.

Theorem 2.7. Let $f(z)=\frac{1}{z}$ and

$$
f_{n}(z)=\frac{\{n(1-B)+1-A\} \frac{1}{z}-(B-A) z^{n}}{\{n(1-B)+1-A\}-(B-A) z_{0}^{n+1}},
$$

$n=1,2,3, \ldots$ Then $h \in T_{M}^{*}\left(A, B, z_{0}\right)$ if and only if it can be expressed in the form

$$
h(z)=\lambda f(z)+\sum_{n=1}^{\infty} \lambda_{n} f_{n}(z)
$$

where $\lambda \geq 0$ and $\lambda+\sum_{n=1}^{\infty} \lambda_{n}=1$.

Proof. Let us suppose that

$$
\begin{aligned}
h(z) & =\lambda f(z)+\sum_{n=1}^{\infty} \lambda_{n} f_{n}(z) \\
& =\frac{a}{z}-\sum_{n=1}^{\infty}\left|a_{n}\right| z^{n}
\end{aligned}
$$

where

$$
a=\lambda+\sum_{n=1}^{\infty} \frac{\{n(1-B)+1-A\} \lambda_{n}}{\{n(1-B)+1-A\}-(B-A) z_{0}^{n+1}}
$$

and

$$
\left|a_{n}\right|+\frac{(B-A) \lambda_{n}}{\{n(1-B)+1-A\}-(B-A) z_{0}^{n+1}},(n=1,2, \ldots) .
$$

Then, it is easy to see that $f\left(z_{0}\right)=\frac{1}{z_{0}}$ and the condition (2.4) is satisfied.

Hence $h \in T_{M}^{*}\left(A, B, z_{0}\right)$.

Conversely let $h \in T_{M}^{*}\left(A, B, z_{0}\right)$, and

$$
h(z)=\frac{a}{z}-\sum_{n=1}^{\infty}\left|a_{n}\right| z^{n} .
$$

Then, from (2.4)

$$
\left|a_{n}\right| \leq \frac{B-A}{\{n(1-B)+1-A\}-(B-A) z_{0}^{n+1}}, \quad(n=1,2,3, \ldots) .
$$

Setting

$$
\lambda_{n}=\left[\frac{\{n(1-B)+1-A\}-(B-A) z_{0}^{n+1}}{(B-A)}\right]\left|a_{n}\right|
$$


and

$$
\lambda=1-\sum_{n=1}^{\infty} \lambda_{n}
$$

we have

$$
h(z)=\lambda f(z)+\sum_{n=1}^{\infty} \lambda_{n} f_{n}(z) .
$$

This completes the proof of theorem.

Theorem 2.9 If $f(z)=\frac{a}{z}-\sum_{n=1}^{\infty}\left|a_{n}\right| z^{n} \in T_{M}^{*}\left(A, B, z_{0}\right)$ and $g(z)=\frac{b}{z}-$ $\sum_{n=1}^{\infty}\left|b_{n}\right| z^{n}$ with $\left|b_{n}\right| \leq 1$ for $n=1,2, \ldots$, then $f * g \in T_{M}^{*}\left(A, B, z_{0}\right)$.

Proof. Let $f(z)=\frac{a}{z}-\sum_{n=1}^{\infty}\left|a_{n}\right| z^{n}$ and $g(z)=\frac{b}{z}-\sum_{n=1}^{\infty}\left|b_{n}\right| z^{n}$, then for convolution of functions $f$ and $g$ we can write

$$
\begin{aligned}
& \sum_{n=1}^{\infty}\left[\{n(1-B)+1-A\}-(B-A) z_{0}^{n+1}\right]\left|a_{n} b_{n}\right| \\
= & \sum_{n=1}^{\infty}\left[\{n(1-B)+1-A\}-(B-A) z_{0}^{n+1}\right]\left|a_{n}\right|\left|b_{n}\right| \\
\leq & \sum_{n=1}^{\infty}\left[\{n(1-B)+1-A\}-(B-A) z_{0}^{n+1}\right]\left|a_{n}\right|, \quad \text { because }\left|b_{n}\right| \leq 1 . \\
\leq & (B-A), \text { by }(2.4) .
\end{aligned}
$$

Hence, by Theorem $2.2, f * g \in T_{M}^{*}\left(A, B, z_{0}\right)$.

Note. It will be of interest to find some other convolution results analogous to those of Juneja and Reddy [2].

\section{Acknowledgement}

The authors are thankfull to the referee for the helpfull suggestions.

\section{Reîerences}

[1] J. Clunie, "On meromorphic schlicht functions," J. Lond. Math. Soc., 34(1959), 215-16.

[2] O.P. Juneja, and T.R. Reddy, "Meromorphic starlike univalent functions with positive coefficients," Annales Universitatis Mariae Curie sklodowska Lubin-Polonia, Vol.XXXIX, 9, Section A, (1985), 55-75.

[3] J. Kaczmarski, "On the coefficients of some class of starlike functions," Bull. Acad, Polon, Sci. Ser. Sci. Math. Astronom. Phys., 17(1969), 495-501.

[4] V. Kumar, "Starlike and convex function with negative and missing coefficients," Jnanabha, 13 (1983), 117-134.

[5] V. Kumar and S.L. Shukla, "Certain classes of univalent functions with negative and missing coefficients," Bulletin of the Institute of Mathematics Academia Sinica, Volume 13, Number 2, June 1985. 
[6] Z. Nehari, Conformal mapping, Dover Publication INC. New York, (1952), Ex. 8, p.238.

[7] Ch. Pommerenke, "On meromorphic starlike functions," Pacific J. Math., 13(1963), 221-235.

[8] W.C. Royster, "Meromorphic starlike multivalent functions," Trans. Amer. Math. Soc., 107(1963), 300-303.

[9] H. Silverman, "Extreme points of univalent functions with two fixed points," Trans. Amer. Math. Soc., 219(1976), 387-395.

[10] H. Silverman and E.M. Silvia, "Fixed coefficients for subclasses of starlike functions," Houston J. Math., 7(1981). 129-136.

[11] H. Silverman, "Univalent functions with negative coefficients," Proc. Amer. Math. Soc., 51(1975), 109-116.

[12] H. Silverman and E.M. Silvia, "Prestarlike functions with negative coefficients," Internat. J. Math. and Math. Scl., 2(1979), 427-439.

[13] E.M. Silvia, Classes related to $\alpha$-starlike functions, Ph.D. Dissertation, Clark University, Worcester, Mass., 1972.

Department of Mathematics, Janta College, Bakewar 206124 Etawah (U.P.), India 